\title{
Harnack Type Inequality: the Method of Moving Planes
}

\author{
Yan Yan $\mathrm{Li}^{\star}$ \\ Department of Mathematics, Rutgers University, Piscataway, NJ 08854-8019, USA. \\ E-mail: yyli@math.rutgers.edu
}

Received: 30 September 1997 / Accepted: 21 July 1998

Abstract: A Harnack type inequality is established for solutions to some semilinear elliptic equations in dimension two. The result is motivated by our approach to the study of some semilinear elliptic equations on compact Riemannian manifolds, which originated from some Chern-Simons Higgs model and have been studied recently by various authors.

\section{Introduction}

Let $(M, g)$ be a compact Riemann surface without boundary, $V$ be a positive function on $M, W$ be a function with $\int_{M} W d v_{g}=1$. Throughout the paper $d v_{g}$ denotes the volume element of $g, \Delta_{g}$ denotes the Laplace Beltrami operator with respect to $g$. For $\lambda \in \mathbb{R}$, we seek a solution of

$$
-\Delta_{g} u=\lambda\left(\frac{V e^{u}}{\int_{M} V e^{u} d v_{g}}-W\right) \quad \text { on } M . \quad\left(E_{u}\right)_{\lambda}
$$

Clearly $\int_{M} W d v_{g}=1$ is a necessary condition for $\left(E_{u}\right)_{\lambda}$ to have a solution. If we set $\xi=u-\log \int_{M} V e^{u} d v_{g}$ for a solution of $\left(E_{u}\right)_{\lambda}$, then $\xi$ satisfies

$$
-\Delta_{g} \xi=\lambda\left(V e^{\xi}-W\right) \quad \text { on } M, \quad\left(E_{\xi}\right)_{\lambda}
$$

and

$$
\int_{M} V e^{\xi} d v_{g}=1
$$

* Partially supported by the Alfred P. Sloan Foundation Research Fellowship, NSF grant DMS-9706887, and a Rutgers University Research Council grant. 
Equation $\left(E_{u}\right)_{\lambda}$ has been studied by Kazdan and Warner [29] in connection with the prescribed Gauss curvature problem, while, it also arises from some Chern-Simons Higgs model as discussed in Taubes [40, 41], Hong, Kim and Pac [27], Jackiw and Weinberg [28], Spruck and Yang [38], Caffarelli and Yang [11], Tarantello [39], Struwe and Tarantello [35], Ding, Jost, Li and Wang [22, 23], and the references therein. Related problems are studied by Carleson and Chang in [14]. Such equations on bounded domains of $\mathbb{R}^{2}$ with Dirichlet boundary conditions play an important role in the context of statistical mechanics of point vortices in the mean field limit as discussed in Caglioti, Lions, Marchioro and Pulvirenti [12,13] and Kiessling [30]. In particular, it is proved in [35], when $(M, g)$ is a flat torus with fundamental cell domain $\left[-\frac{1}{2}, \frac{1}{2}\right] \times\left[-\frac{1}{2}, \frac{1}{2}\right]$, $V \equiv 1$ and $W \equiv 1 / \operatorname{vol}(M)$, that Eq. $\left(E_{u}\right)_{\lambda}$ has at least one nontrivial solution for $8 \pi<\lambda<4 \pi^{2}$. On the other hand Eq. $\left(E_{u}\right)_{8 \pi}$, with $W \equiv 1 / \operatorname{vol}(M)$, is studied in [22] where sufficient conditions are given for the existence of solutions. Such conditions obviously hold when $(M, g)$ is a flat two dimensional torus, $V \equiv 1$ and $W \equiv 1 / \operatorname{vol}(M)$. The author was recently informed by G. Tarantello that she and M. Nolasco have independently established the existence results in the special case that $(M, g)$ is a flat two-dimensional torus, $V \equiv 1$ and $W \equiv 1 / \operatorname{vol}(M)$.

In view of our earlier work [31], we propose a different approach to study the existence of solutions of $\left(E_{u}\right)_{\lambda}$. Clearly $\left(E_{u}\right)_{\lambda}$ is invariant when replacing $u$ by $u+$ constant. Assuming $V$ and $W$ are Lipschitz functions, it is well known that when $\lambda$ lies in compact subsets of $(-\infty, 8 \pi)$, all solutions $u$ of $\left(E_{u}\right)_{\lambda}$, after a normalization $\int_{M} u d v_{g}=0$, stay bounded in $C^{2, \alpha}(M)$ for $0<\alpha<1$. For $\lambda$ in compact subsets of $\cup_{m=1}^{\infty}(8 \pi m, 8 \pi(m+1))$, the same conclusion holds due to the results of Brezis and Merle [8] and Li and Shafrir [32]. For $0<\alpha<1$, let

$$
X_{\alpha}=\left\{u \in C^{2, \alpha}(M) \mid \int_{M} u d v_{g}=0\right\} .
$$

$X_{\alpha}$, equipped with the $C^{2, \alpha}(M)$ norm, is a Banach space. We introduce an operator $K_{\lambda}: X_{\alpha} \rightarrow X_{\alpha}$ by

$$
K_{\lambda}(u)=\lambda\left(-\Delta_{g}\right)^{-1}\left(\frac{V e^{u}}{\int_{M} V e^{u} d v_{g}}-W\right) .
$$

It follows from standard elliptic theories that $K_{\lambda}$ is a well defined compact operator. Equation $\left(E_{u}\right)_{\lambda}$ is equivalent to $\left(I-K_{\lambda}\right) u=0$ in $X_{\alpha}$. For any bounded open set $O \subset X_{\alpha}$, the Leray-Schauder degree $\operatorname{deg}\left(I-K_{\lambda}, O, 0\right)$ is well defined provided 0 does not belong to $\left(I-K_{\lambda}\right)(\partial O)$. For the definition of the Leray-Schauder degree and its various properties, see, for example, Nirenberg [34]. Let

$$
B_{a}=\left\{u \in X_{\alpha} \mid\|u\|_{X_{\alpha}}<a\right\}
$$

denote the ball in $X_{\alpha}$. Due to the above mentioned a priori estimates of solutions of $\left(E_{u}\right)_{\lambda}$ for $\lambda$ in compact subsets of $\mathbb{R} \backslash \cup_{m=1}^{\infty}\{8 \pi m\}$, there exists some continuous function $a_{\lambda}$ defined in $\mathbb{R} \backslash \cup_{m=1}^{\infty}\{8 \pi m\}$ such that for all $\lambda \in \mathbb{R} \backslash \cup_{m=1}^{\infty}\{8 \pi m\}$ and $a>a_{\lambda}$,

$$
d_{\lambda}:=\operatorname{deg}\left(I-K_{\lambda}, B_{a}, 0\right)
$$

is well defined and, in view of the homotopy invariance of the Leray-Schauder degree, is independent of $a$ as long as $a>a_{\lambda}$. Moreover $d_{\lambda}$ is a constant in each interval $(8 \pi m, 8 \pi(m+1))$. The piecewise constant function $d_{\lambda}$ is determined by the Euler number of $M$. We know that $d_{\lambda}$ is equal to 1 for $\lambda<8 \pi$. However, due to the possible loss of 
compactness of solutions of $\left(E_{u}\right)_{\lambda}$ when $\lambda$ crosses $8 \pi m$, we do not know yet the values of $d_{\lambda}$ in the other intervals. Knowing the values of $d_{\lambda}$ should lead to new existence results for $\left(E_{u}\right)_{\lambda}$ since $d_{\lambda} \neq 0$ implies that $\left(E_{u}\right)_{\lambda}$ has at least one solution. The situation here is similar to that in [31] where

$$
-\Delta u+2 u=\frac{1}{6} K(x) u^{3}, \quad u>0, \quad \text { on } \mathbb{S}^{4}
$$

is studied. Let $\mathcal{A}$ be the open and dense subset of $C^{2}\left(\mathbb{S}^{4}\right)^{+}$, the set of positive twice differentiable functions, defined in [31]. For any Morse function $K \in \mathcal{A}$, let $K_{\lambda}=$ $(1-\lambda)+\lambda K$ for $0 \leq \lambda \leq 1$. It is not difficult to see from [31] that there exist $0<\lambda_{1}<\cdots<\lambda_{l}<1$ such that for all $\lambda \in(0,1] \backslash\left\{\lambda_{1}, \cdots, \lambda_{l}\right\}$ the total LeraySchauder degree $d_{\lambda}$ of all possible solutions of (3) with $K=K_{\lambda}$ is well defined and is a constant function of $\lambda$ in each interval $\left(\lambda_{m}, \lambda_{m+1}\right)$. Since $d_{1} \neq 0$ implies that (3) has at least one solution, we wish to have a formula of $d_{1}$ in terms of $K$. The formula of $d_{\lambda}$ for small $\lambda$ is known due to the work of Chang and Yang [16]. So, one way to derive a formula of $d_{1}$ is to calculate the jump-values of $d_{\lambda}$ at $\lambda_{m}$ for $1 \leq m \leq l$. These jump-values can be calculated by using the strong pointwise estimates in [31] of blowup solutions $u_{\lambda}$, solutions of (3) with $K=K_{\lambda}$, as $\lambda \rightarrow \lambda_{m}$. Once these jump-values are known, we have a formula of $d_{1}$ in terms of $K$. This provides an alternative derivation of the formula of $d_{1}$ obtained in [31].

We propose to take a similar approach to study $\left(E_{u}\right)_{\lambda}$, namely, to look for a formula of $d_{\lambda}$ in terms of the Euler number of $M$. Since we know $d_{\lambda}=1$ for $\lambda<8 \pi$, we only need to calculate the jump-values of $d_{\lambda}$ at $8 \pi m, m \geq 1$. In view of the results in [31], we tend to believe that a good enough pointwise estimate of blowup solutions $\left\{u_{\lambda}\right\}$ as $\lambda \rightarrow 8 \pi m$ is the most crucial step in evaluating the jump-value of $d_{\lambda}$ at $8 \pi m$. Once we know the jump-values for $m$ less than some $m_{0}$, we obtain a formula of $d_{\lambda}$ in $\left(-\infty, 8 \pi m_{0}\right) \backslash \cup_{m=1}^{m_{0}-1}\{8 \pi m\}$. The main purpose of this paper is to start making good pointwise estimates for blowup solutions $\left\{u_{\lambda}\right\}$ as $\lambda \rightarrow 8 \pi m$. The main analytical result of this paper is a new local estimate given in Theorem 0.3.

We first state a well known fact in the subcritical case $\lambda<8 \pi$.

Theorem 0.1 (well known). Let $(M, g)$ be a compact Riemann surface, $V$ be a positive continuous function on $M, W \in L^{\infty}(M)$ with $\int_{M} W d v_{g}=1$. Then for all $\epsilon>0$, $-\epsilon^{-1} \leq \lambda \leq 8 \pi-\epsilon$, and all $C^{2}$ solutions $u$ of $\left(E_{u}\right)_{\lambda}$ with $\int_{M} u d v_{g}=0$, we have

$$
\|u\|_{L^{\infty}(M)} \leq C,
$$

where $C$ depends only on $M, g, \epsilon,\|V\|_{L^{\infty}(M)},\|W\|_{L^{\infty}(M)}$, the modulo of continuity of $V$, and the positive lower bound of $V$.

If both $V$ and $W$ are Lipschitz functions, then it is well known that any $C^{2}$ solution of $\left(E_{u}\right)_{\lambda}$ is actually in $C^{2, \alpha}(M)$ for all $0<\alpha<1$, and $C^{2, \alpha}$ estimates of $u$ follow from the $L^{\infty}$ estimates. Thus, we have

Corollary 0.1. In addition to the hypothesis in Theorem 0.1, assume that both $V$ and $W$ are Lipschitz functions. Then for all $\epsilon>0,-\epsilon^{-1} \leq \lambda \leq 8 \pi-\epsilon, 0<\alpha<1$, and all $C^{2}$ solution $u$ of $\left(E_{u}\right)_{\lambda}$ with $\int_{M} u d v_{g}=0$, we have

$$
\|u\|_{C^{2, \alpha}(M)} \leq C
$$

where $C$ depends only on $M, g, \epsilon, \alpha,\|V\|_{L^{\infty}(M)},\|\nabla V\|_{L^{\infty}(M)},\|\nabla W\|_{L^{\infty}(M)}$, and the positive lower bound of $V$. 
Corollary 0.2. In addition to the hypothesis in Theorem 0.1, we assume that both $V$ and $W$ are Lipschitz functions. Then

$$
d_{\lambda}=1
$$

for all $\lambda<8 \pi$. Consequently, $\left(E_{u}\right)_{\lambda}$ has at least one solution for every $\lambda<8 \pi$.

Remark 0.1. The existence of one solution to $\left(E_{u}\right)_{\lambda}$ for $\lambda<8 \pi$ can easily be established by variational methods using the following consequence of the Moser-Trudinger inequality: For every $\epsilon>0$,

$\log \int_{M} e^{w} d v_{g} \leq\left(\frac{1}{16 \pi}+\epsilon\right) \int_{M}\left|\nabla_{g} w\right|^{2} d v_{g}+\frac{1}{\operatorname{vol}(M)} \int_{M} w d v_{g}+C(\epsilon) \quad \forall w \in H^{1}(M)$.

See, for example, [33, 29] and [22] for more details.

For $\lambda \geq 8 \pi$, Eq. $\left(E_{u}\right)_{\lambda}$ is much more delicate. The difficulty lies in possible loss of compactness of solutions of $\left(E_{u}\right)_{\lambda}$ for $\lambda \geq 8 \pi$. A good understanding of possible blowup behavior of solutions of $\left(E_{u}\right)_{\lambda}$ is important in the study of $\left(E_{u}\right)_{\lambda}$. Our next theorem gives some understanding of the possible blowup behavior of solutions of $\left(E_{u}\right)_{\lambda}$, which should be relevant in the study of the existence of solutions of $\left(E_{u}\right)_{\lambda}$ for $\lambda \geq 8 \pi$.

Let $\left\{V_{n}\right\}$ satisfy

$$
\liminf _{n \rightarrow \infty} \min _{M} V_{n}>0, \quad \limsup _{n \rightarrow \infty}\left(\max _{M} V_{n}+\left\|\nabla V_{n}\right\|_{L^{\infty}(M)}\right)<\infty,
$$

$\left\{W_{n}\right\}$ satisfy

$$
\limsup _{n \rightarrow \infty}\left\|\nabla W_{n}\right\|_{L^{\infty}(M)}<\infty, \int_{M} W_{n} d v_{g}=1,
$$

and $\left\{\xi_{n}\right\}$ satisfy

$$
-\Delta_{g} \xi_{n}=\lambda_{n}\left(V_{n} e^{\xi_{n}}-W_{n}\right) \quad \text { on } M
$$

and

$$
\int_{M} V_{n} e^{\xi_{n}} d v_{g}=1
$$

We will use $d(x, y)$ to denote the distance between $x$ and $y$ in $M$ and will use the notation

$$
\bar{\xi}_{n}=\frac{1}{\operatorname{vol}(M)} \int_{M} \xi_{n} d v_{g}
$$

to denote the average of $\xi_{n}$ on $M$.

Let $G(x, y)$ denote the Green's function of $-\Delta_{g}$ on $M$, namely,

$$
\left\{\begin{aligned}
-\Delta_{x} G(x, y) & =\delta_{y}-\frac{1}{\operatorname{vol}(M)}, \text { in } M, \\
\int_{M} G(x, y) d v_{g}(x) & =0 .
\end{aligned}\right.
$$

It is well known (see, e.g., [2]) that $G(x, y)$ is uniquely defined, symmetric in $x$ and $y$, and a solution of (6) satisfies

$$
\xi_{n}(x)-\bar{\xi}_{n}=\lambda_{n} \int_{M}\left(V_{n}(y) e^{\xi_{n}(y)}-W_{n}(y)\right) G(x, y) d v_{g}(y), \quad \forall x \in M .
$$


Theorem 0.2. Let $(M, g)$ be a compact Riemann surface, $\left\{V_{n}\right\}$ and $\left\{W_{n}\right\}$ satisfy (4) and (5), $\lambda_{n} \rightarrow \bar{\lambda} \in(-\infty, \infty)$, and $\left\{\xi_{n}\right\} \subset C^{2}(M)$ satisfy (6) and (7). Assume

$$
\max _{M}\left|\xi_{n}\right| \rightarrow \infty
$$

Then after passing to a subsequence (still denoted as $\left\{\xi_{n}\right\}$ ), there exist $m$ distinct points $\left\{\bar{x}^{(l)}\right\}_{1 \leq l \leq m}$ in $M$ and $m$ sequences of points $\bar{x}_{n}^{(l)} \rightarrow \bar{x}^{(l)}$ such that

(a) $\xi_{n} \rightarrow-\infty$ uniformly on compact subsets of $M \backslash\left\{\bar{x}^{(1)}, \cdots, \bar{x}^{(m)}\right\}$.

(b) For each $1 \leq l \leq m$, and $n$ large, $\bar{x}_{n}^{(l)}$ is the unique maximum point of $\xi_{n}$ in $\left\{x \in M \mid d\left(x, \bar{x}^{(l)}\right) \leq \frac{1}{2} \min _{l^{\prime} \neq l} \operatorname{dist}\left(\bar{x}^{\left(l^{\prime}\right)}, \bar{x}^{(l)}\right)\right\}$, and $\xi_{n}\left(\bar{x}_{n}^{(l)}\right) \rightarrow \infty$.

(c) For each $1 \leq l \leq m$, let $g=e^{\varphi_{n}}\left(d x_{1}^{2}+d x_{2}^{2}\right)$ be an isothermal coordinate system (with $\varphi_{n}(0)=0$ ) centered at $\bar{x}_{n}^{(l)}$, we have, for some constant $C$ independent of $n$,

$$
\left|\xi_{n}(x)-\log \frac{e^{\xi_{n}(0)}}{\left(1+\frac{\lambda_{n} V_{n}(0)}{8} e^{\xi_{n}(0)}|x|^{2}\right)^{2}}\right| \leq C, \quad \forall|x| \leq \frac{1}{4} \min _{l^{\prime} \neq l} \operatorname{dist}\left(\bar{x}^{(l)}, \bar{x}^{\left(l^{\prime}\right)}\right) \text { and } \forall n .
$$

(d) For some constant $C$ independent of $n$,

$$
\max _{1 \leq l \leq m}\left|\xi_{n}\left(x_{n}^{(l)}\right)+\bar{\xi}_{n}\right| \leq C
$$

(e) In $C_{l o c}^{2}\left(M \backslash\left\{\bar{x}^{(1)}, \cdots, \bar{x}^{(m)}\right\}\right)$,

$$
\xi_{n}-\bar{\xi}_{n} \rightarrow 8 \pi \sum_{l=1}^{m} G\left(\cdot, \bar{x}^{(l)}\right)-8 \pi m \int_{M} W(y) G(\cdot, y) d v_{g}(y),
$$

where $W=\lim _{n \rightarrow \infty} W_{n}$ weak $*$ in $L^{\infty}(M)$. Consequently,

$$
\lambda_{n} V_{n} e^{\xi_{n}} \rightarrow 8 \pi \sum_{l=1}^{m} \delta_{\bar{x}^{(l)}} \text { in the sense of measure, and } \bar{\lambda}=8 \pi m,
$$

where $\delta_{\bar{x}^{(l)}}$ denotes the Delta mass at $\bar{x}^{(l)}$.

Remark 0.2. Theorem 0.2 still holds when we replace the metric $g$ by a sequence of metrics $g_{n}$ converging to $g$ in the $C^{2}$ norm. This can be seen easily from the proof of Theorem 0.2.

Due to Theorem 0.2 and Theorem 0.1 , there exists some continuous function $a_{\lambda}$ defined in $\mathbb{R} \backslash \cup_{m=1}^{\infty}\{8 \pi m\}$ such that $d_{\lambda}$ in (2) is well defined for all $\lambda \in \mathbb{R} \backslash \cup_{m=1}^{\infty}\{8 \pi m\}$ and $a>a_{\lambda}$. Furthermore, in view of Remark $0.2, d_{\lambda}$ is independent of the metric $g$. Therefore, in view of the homotopy invariance of the Leray-Schauder degree, $d_{\lambda}$ is a constant in each of the open intervals, and all these constants are independent of $V, W$ and the metric $g$. So $d_{\lambda}$ is a piecewise constant function of $\lambda$ determined completely by the Euler number of $M$. We know from Corollary 0.2 that $d_{\lambda}$ is equal to 1 for $\lambda<8 \pi$, but we do not know yet the values of $d_{\lambda}$ in other intervals. Knowing the values of $d_{\lambda}$ should lead to new existence results for $\left(E_{u}\right)_{\lambda}$. As mentioned earlier we wish to calculate the jump-value of $d_{\lambda}$ at $8 \pi m$. In view of the results in [31], Theorem 0.2, providing pointwise estimates of $\left\{\xi_{n}\right\}$, should be useful in evaluating the jump-value of $d_{\lambda}$ at $8 \pi m$.

Let $\left\{\xi_{n}\right\}$ be the subsequence in Theorem 0.2 satisfying (a)-(e). In an isothermal coordinate system centered at $\bar{x}_{n}^{(l)}$, we set 


$$
v_{n}(x)=\xi_{n}\left(\delta_{n}^{(l)} x\right)+2 \log \delta_{n}^{(l)}, \quad|x|<a / \delta_{n}^{(l)},
$$

where $\delta_{n}^{(l)}=e^{-\xi_{n}\left(\bar{x}_{n}^{(l)}\right) / 2}$ and $a$ is some suitably small positive constant. It will be shown by a blow up argument that

$$
v_{n} \rightarrow v \quad \text { in } C_{l o c}^{2}\left(\mathbb{R}^{2}\right)
$$

with

$$
v(x)=\log \left\{\frac{1}{\left(1+\frac{\bar{\lambda}\left(\lim _{n \rightarrow \infty} V_{n}(0)\right.}{8}|x|^{2}\right)^{2}}\right\}, \quad \text { in } \mathbb{R}^{2} .
$$

Consequently,

$$
\bar{R}_{n}^{(l)}:=\sup \left\{R>0:\left\|v_{n}-v\right\|_{C^{2}\left(\bar{B}_{2 R}(0)\right)}+\left\|v_{n}-v\right\|_{H^{2}\left(\bar{B}_{2 R}(0)\right)}<e^{-R}\right\} \rightarrow \infty .
$$

This shows that $\xi_{n}(x)$ is very well approximated by $\log \left\{\frac{e^{\xi_{n}\left(\bar{x}_{n}^{(l)}\right)}}{\left(1+\frac{\lambda_{n} V_{n}(0)}{8} e^{\xi_{n}\left(\bar{x}_{n}^{(l)}\right)}|x|^{2}\right)^{2}}\right\}$ in $|x| \leq \bar{R}_{n}^{(l)} \delta_{n}^{(l)}$. For $\bar{R}_{n}^{(l)} \delta_{n}^{(l)} \leq|x| \leq \frac{1}{2} \min _{l^{\prime} \neq l} \operatorname{dist}\left(\bar{x}^{\left(l^{\prime}\right)}, \bar{x}^{(l)}\right)$, we will give, using (c), some convergence estimate better than (e). For convenience, we use the notation

$$
\zeta_{n} \sim 0
$$

to denote a sequence of functions $\left\{\zeta_{n}\right\}$ in $C^{2}(M)$ satisfying

$$
\begin{gathered}
\lim _{n \rightarrow \infty} \max \left\{\frac{\left|\zeta_{n}(x)\right|}{1+\sum_{j=1}^{m}\left|\log d\left(x, \bar{x}_{n}^{(j)}\right)\right|}: x \in M \backslash \cup_{l=1}^{l} B_{\bar{R}_{n}^{(l)} \delta_{n}^{(l)}}\left(\bar{x}_{n}^{(l)}\right)\right\}=0, \\
\lim _{n \rightarrow \infty} \max \left\{\frac{\left|\nabla \zeta_{n}(x)\right|}{\sum_{j=1}^{m} d\left(x, \bar{x}_{n}^{(j)}\right)^{-1}}: x \in M \backslash \cup_{l=1}^{l} B_{\bar{R}_{n}^{(l)} \delta_{n}^{(l)}}\left(\bar{x}_{n}^{(l)}\right)\right\}=0,
\end{gathered}
$$

and

$$
\lim _{n \rightarrow \infty} \max \left\{\frac{\left|\nabla^{2} \zeta_{n}(x)\right|}{\sum_{j=1}^{m} d\left(x, \bar{x}_{n}^{(j)}\right)^{-2}}: x \in M \backslash \cup_{l=1}^{l} B_{\bar{R}_{n}^{(l)} \delta_{n}^{(l)}}\left(\bar{x}_{n}^{(l)}\right)\right\}=0 .
$$

We also write $\zeta \sim_{0} 0$ for (11), $\zeta \sim_{1} 0$ for (12), and $\zeta \sim_{2} 0$ for (13).

Corollary 0.3. Let $\left\{\xi_{n}\right\}$ be the subsequence in Theorem 0.2 satisfying (a)-(e). Then

$$
\varphi_{n}:=\xi_{n}-\bar{\xi}_{n}-8 \pi \sum_{l=1}^{m} G\left(\cdot, \bar{x}_{n}^{(l)}\right)+8 \pi m \int_{M} W(y) G(\cdot, y) d v_{g}(y) \sim 0 .
$$

Theorem 0.2 will be deduced from some local results on the behavior of blowup solutions to equations of the type $-\Delta u=V e^{u}$ in domains of $\mathbb{R}^{2}$. In particular, a new local estimate, Theorem 0.3 , is needed in the proof of Theorem 0.2 . We first recall some known results.

Let $\Omega \subset \mathbb{R}^{2}$ be a bounded smooth domain, $0 \in \Omega,\left\{V_{n}\right\}$ be a sequence of Lipschitz continuous functions satisfying

$$
0<a \leq V_{n}(x) \leq b<\infty, \quad \forall x \in \bar{\Omega},
$$


and

$$
\left|\nabla V_{n}(x)\right| \leq A, \quad \forall x \in \bar{\Omega},
$$

where $a, b$ and $A$ are positive constants.

Consider

$$
-\Delta u_{n}=V_{n} e^{u_{n}}, \quad \text { in } \Omega,
$$

and let $\left\{u_{n}\right\}$ be a sequence of $C^{2}$ solutions of (16) satisfying

$$
\limsup _{n \rightarrow \infty} \int_{\Omega} V_{n} e^{u_{n}}<\infty .
$$

It follows from Theorem 3 in Brezis and Merle [8] that, under (14)-(17), there are only three alternatives after passing to a subsequence:

1. $\left\{u_{n}\right\}$ uniformly converges on compact subsets of $\Omega$,

2. $\left\{u_{n}\right\}$ tends to $-\infty$ uniformly on compact subsets of $\Omega$,

3. There exist finitely many blowup points $\left\{x^{(1)}, \cdots, x^{(l)}\right\}$ of $\left\{u_{n}\right\}$ such that $\left\{u_{n}\right\}$ tends to $-\infty$ uniformly on compact subsets of $\Omega \backslash\left\{x^{(1)}, \cdots, x^{(l)}\right\}$, and

$$
V_{n} e^{u_{n}}-\sum_{i=1}^{l} \alpha_{i} \delta_{x^{(i)}} \quad \text { in the sense of measure, }
$$

with $\alpha_{i} \geq 4 \pi$. Here $\delta_{x^{(i)}}$ is the Dirac mass at $x^{(i)}$.

We recall that a point $\bar{y}$ is called a blowup point of $\left\{u_{n}\right\}$ if there exist $y_{n} \rightarrow \bar{y}$ such that $u_{n}\left(y_{n}\right) \rightarrow \infty$ as $n \rightarrow \infty$.

It was conjectured in [8] that each $\alpha_{i}$ can be written as $\alpha_{i}=8 \pi m_{i}$ for some positive integer $m_{i}$. This was established by Li and Shafrir in [32]. Chen further demonstrated in [20] that any positive integer $m_{i}$ can occur in such local situations.

Under (14) and (15), the following Harnack type inequality is proved by Brezis, $\mathrm{Li}$ and Shafrir in [9] through the method of moving planes: Every solution of (16) satisfies, on any compact subset $K$ of $\Omega$,

$$
\sup _{K} u_{n}+\inf _{\Omega} u_{n} \leq C(a, b, A, K, \Omega) .
$$

It is raised as an open question in [9] whether the above Harnack type inequality still holds when replacing $\left\|\nabla V_{n}\right\|_{L^{\infty}(\Omega)}$ by $\left\|V_{n}\right\|_{C^{\alpha}(\Omega)}(0<\alpha<1)$. The answer is affirmative due to some recent work of Chen and Lin [19].

Now we are ready to state our new local estimate which is essentially equivalent to a Harnack type estimate $\left|\sup u_{n}+\inf u_{n}\right| \leq C$ under additional hypotheses (20) and (21) below. These additional hypotheses are necessary for such an estimate to hold. We will further assume that

$$
u_{n}(0)=\max _{\bar{\Omega}} u_{n} \rightarrow \infty
$$

and

$$
V_{n} e^{u_{n}} \rightarrow \alpha \delta, \quad \text { in } \bar{\Omega}, \quad \text { in the sense of measure, }
$$

where $\alpha>0$ is a constant and $\delta$ is the Dirac mass at the origin. 
Theorem 0.3. In addition to (14)-(16) and (19)-(20), we assume that

$$
\max _{\partial \Omega} u_{n}-\min _{\partial \Omega} u_{n} \leq A_{1}
$$

for some positive constant $A_{1}$. Then for some constant $C$ independent of $n$, we have

$$
\left|u_{n}(x)-\log \frac{e^{u_{n}(0)}}{\left(1+\frac{V_{n}(0)}{8} e^{u_{n}(0)}|x|^{2}\right)^{2}}\right| \leq C, \quad \forall x \in \bar{\Omega} \text { and } \forall n .
$$

Theorem 0.3 will be proved by the method of moving planes, which has become a very powerful and convenient tool in the study of nonlinear elliptic partial differential equations starting from the pioneering works of A.D. Alexandrov [1], Serrin [37], and Gidas, Ni and Nirenberg [25, 26]. The method has been further developed in a series of papers by Berestycki, Nirenberg and their collaborators [3]-[7], and Caffarelli, Gidas and Spruck [10]. Many more applications of the moving plane method have been given by various authors. The method of moving planes was used to obtain some Harnack type inequalities by Schoen in [36], subsequently by Brezis, Li, and Shafrir in [9], and by Chen and Lin in [18]. Our proof of Theorem 0.3 requires some new ingredients.

\section{Compactness and Existence for $\lambda \in(-\infty, 8 \pi)$}

Throughout this section $V$ is a positive continuous function on $M$ and $W \in L^{\infty}(M)$ with $\int_{M} W d v_{g}=1$.

Lemma 1.1. Let $\epsilon>0$ and $\xi$ satisfy $\left(E_{\xi}\right)_{\lambda}$ and (1) with $-\epsilon^{-1} \leq \lambda \leq 8 \pi-\epsilon$. Then

$$
\max _{M}|\xi| \leq C
$$

for some constant $C$ depending only on $M, g, \epsilon,\|W\|_{L^{\infty}(M)},\|V\|_{L^{\infty}(M)}$, the positive lower bound of $V$, and the modulus of continuity of $V$.

Proof. Suppose the contrary, then there exist $\left\{V_{n}\right\}$ converging to some positive function in $C(M),\left\{W_{n}\right\}$ bounded in $L^{\infty}(M), \lambda_{n} \rightarrow \bar{\lambda} \in\left[-\epsilon^{-1}, 8 \pi-\epsilon\right], \xi_{n}$, with $\int_{M} V_{n} e^{\xi_{n}} d v_{g}=1$, satisfying $\left(E_{\xi}\right)_{\lambda_{n}}$ with $V=V_{n}$ and $W=W_{n}$, but $\max _{M}\left|\xi_{n}\right| \rightarrow \infty$.

Let $y_{n}$ be a maximum point of $\xi_{n}$. If $\xi_{n}\left(y_{n}\right) \rightarrow \infty$ along a subsequence (still denoted as $\left.\left\{\xi_{n}\right\}\right)$, we work in some isothermal coordinate system $x=\left(x_{1}, x_{2}\right)$ centered at $y_{n}$. Without loss of generality, we may assume $y_{n} \rightarrow \bar{y}$. In a neighborhood of $\bar{y}$, $g=e^{\varphi_{n}}\left(d x_{1}^{2}+d x_{2}^{2}\right)$, where $\varphi_{n}(0)=1$ and $\left\{\varphi_{n}\right\}$ converges in the neighborhood with respect to $C^{2}$ norms, and, in the neighborhood, the equation of $\xi_{n}$ takes the form

$$
-\Delta \xi_{n}=\lambda_{n}\left(V_{n} e^{\varphi_{n}} e^{\xi_{n}}-e^{\varphi_{n}} W_{n}\right),
$$

where $\Delta=\partial_{x_{1} x_{1}}+\partial_{x_{2} x_{2}}$. Consider

$$
v_{n}(x)=\xi_{n}\left(\delta_{n} x\right)+2 \log \delta_{n}, \quad|x|<a \delta_{n}^{-1},
$$

where $\delta_{n}=e^{-\xi_{n}(0) / 2} \rightarrow 0$ and $a>0$ is some constant. Clearly $v_{n}$ satisfies

$$
\begin{cases}-\Delta v_{n}(x)=\lambda_{n}\left(V_{n}\left(\delta_{n} x\right) e^{\varphi_{n}\left(\delta_{n} x\right)} e^{v_{n}(x)}-\delta_{n}^{2} e^{\varphi_{n}\left(\delta_{n} x\right)} W_{n}\left(\delta_{n} x\right)\right), & |x|<a \delta_{n}^{-1}, \\ \int_{|x| \leq a \delta_{n}^{-1}} V_{n}\left(\delta_{n} x\right) e^{\varphi_{n}\left(\delta_{n} x\right)} e^{v_{n}(x)} \leq 1, & |x|<a \delta_{n}^{-1} \\ v_{n}(x) \leq v_{n}(0)=0, & \end{cases}
$$


For any $R>1$, let $f_{n}$ be the solution of

$$
\begin{cases}-\Delta f_{n}(x)=\lambda_{n}\left(V_{n}\left(\delta_{n} x\right) e^{\varphi_{n}\left(\delta_{n} x\right)} e^{v_{n}(x)}-\delta_{n}^{2} e^{\varphi_{n}\left(\delta_{n} x\right)} W_{n}\left(\delta_{n} x\right)\right) & ,|x|<R \\ f_{n}(x)=0, & |x|=R .\end{cases}
$$

Then $\left|f_{n}\right|$ is bounded from above by some constant $C=C(R)$ in $|x| \leq R$, so $C+f_{n}-v_{n}$ is a nonnegative harmonic function in $|x| \leq R$ with value at the origin not larger than $2 C$. The Harnack inequality yields the upper bound of $C+f_{n}-v_{n}$ in $|x| \leq R / 2$, which in turn yields the lower bound of $v_{n}$ in $|x| \leq R / 2$. Therefore, after passing to a subsequence, we have, by applying $W^{2, p}$ estimates to $v_{n}$, that

$$
v_{n} \rightarrow v \quad \text { in } C_{l o c}^{1}\left(\mathbb{R}^{2}\right)
$$

where $v$ satisfies, in the distribution sense,

$$
\left\{\begin{array}{l}
-\Delta v=\bar{\lambda}\left(\lim _{n \rightarrow \infty} V_{n}(0)\right) e^{v}, \text { in } \mathbb{R}^{2}, \\
\left(\lim _{n \rightarrow \infty} V_{n}(0)\right) \int_{\mathbb{R}^{2}} e^{v} \leq 1, \\
v(x) \leq v(0)=0, \quad \text { in } \mathbb{R}^{2} .
\end{array}\right.
$$

In fact, due to standard elliptic estimates, $v \in C^{2}\left(\mathbb{R}^{2}\right)$.

It is easy to show (see the Appendix) that there is no solution to (23) if $\bar{\lambda} \leq 0$, so $\bar{\lambda}>0$. On the other hand, due to the classification of all solutions of (23) (see, for example, $[19,21]$ and $[15])$, we know that $v$ is the function given in (10). It follows that

$$
\bar{\lambda}\left(\lim _{n \rightarrow \infty} V_{n}(0)\right) \int_{\mathbb{R}^{2}} e^{v}=8 \pi
$$

Consequently, $\bar{\lambda} \geq 8 \pi$. This is a contradiction. Thus $\left\{\xi_{n}\right\}$ is bounded from above and $\xi_{n}\left(\hat{y}_{n}\right)=-\min _{M} \xi_{n} \rightarrow \infty$ for some $\hat{y}_{n} \in M$. Without loss of generality, we may assume $\hat{y}_{n} \rightarrow \hat{y}$. Let $\Omega \subset M$ be any smooth open connected set containing $\hat{y}, \partial \Omega \neq \phi$. Define $\eta_{n}$ by

$$
\begin{cases}-\Delta_{g} \eta_{n}=\lambda_{n}\left(V_{n} e^{\xi_{n}}-W_{n}\right), & \text { in } \Omega, \\ \eta_{n}=0, & \text { on } \partial \Omega .\end{cases}
$$

In view of the upper bound of $\xi_{n}$, we derive from standard elliptic estimates that $\left\{\eta_{n}\right\}$ is uniformly bounded in $\bar{\Omega}$. Let $w_{n}=\xi_{n}-\eta_{n}$, then $w_{n}$ satisfies

$$
-\Delta_{g} w_{n}=0, \quad w_{n} \leq C, \quad \text { in } \Omega \text {. }
$$

Applying the Harnack inequality to $C-w_{n}$ on compact subsets of $\Omega$, we have, in view of $C-w_{n}\left(\hat{y}_{n}\right) \rightarrow \infty$, that $C-w_{n} \rightarrow \infty$ uniformly on compact subsets of $\Omega$. Namely, $\xi_{n} \rightarrow-\infty$ uniformly on compact subsets of $\Omega$. Since $\Omega$ can be chosen arbitrarily, $\xi_{n} \rightarrow-\infty$ uniformly on $M$ which violates $\int_{M} V_{n} e^{\xi_{n}}=1$. Lemma 1.1 is established.

Theorem 0.1 can be deduced from Lemma 1.1 as follows.

Proof of Theorem 0.1. Set $\xi=u-\log \int_{M} V e^{u} d v_{g}$. We know from Lemma 1.1 that $|\xi| \leq C$ on $M$. Since $\int_{M} u d v_{g}=0, u$ vanishes somewhere in $M$. It follows that $\left|\log \int_{M} V e^{u} d v_{g}\right| \leq C$. Consequently, $|u| \leq C$. 


\section{A New Local Estimate by the Method of Moving Planes}

In this section we establish Theorem 0.3 by the method of moving planes.

Let $\bar{G}(x, y)$ be the Green's function of $-\Delta$ in $\Omega \subset \mathbb{R}^{2}$ with respect to the zero boundary condition:

$$
\left\{\begin{aligned}
-\Delta_{x} \bar{G}(x, y) & =\delta_{y}, \text { in } \Omega \\
\bar{G}(x, y) & =0, \quad x \in \partial \Omega
\end{aligned}\right.
$$

Consider

$$
\tilde{u}_{n}(x)=\int_{\Omega} \bar{G}(x, y) V_{n}(y) e^{u_{n}(y)} d y
$$

Namely, $\tilde{u}_{n}$ is the solution of

$$
\begin{cases}-\Delta \tilde{u}_{n}=V_{n} e^{u_{n}}, & \text { in } \Omega \\ \tilde{u}_{n}=0, & \text { on } \partial \Omega\end{cases}
$$

Lemma 2.1. Under the hypothesis of Theorem 0.3, for all $r>0$,

$$
\tilde{u}_{n}(x) \rightarrow \alpha \bar{G}(x, 0) \quad \text { in } C^{1}\left(\bar{\Omega} \backslash B_{r}\right)
$$

Proof. Write

$$
\tilde{u}_{n}(x)=\bar{G}(x, 0) \int_{\Omega} V_{n}(y) e^{u_{n}(y)} d y+\int_{\Omega}[\bar{G}(x, y)-\bar{G}(x, 0)] V_{n}(y) e^{u_{n}(y)} d y
$$

As $y \rightarrow 0, \bar{G}(x, y)-\bar{G}(x, 0) \rightarrow 0$ uniformly for $x \in \bar{\Omega} \backslash B_{r}$. Consequently, using (20),

$$
\tilde{u}_{n}(x) \rightarrow \alpha \bar{G}(x, 0) \quad \text { in } C^{0}\left(\bar{\Omega} \backslash B_{r}\right) .
$$

On the other hand, we have

$$
\nabla \tilde{u}_{n}(x)=\int_{\Omega} \nabla_{x} \bar{G}(x, y) V_{n}(y) e^{u_{n}(y)} d y,
$$

and, as $y \rightarrow 0, \nabla_{x} \bar{G}(x, y)-\nabla_{x} \bar{G}(x, 0) \rightarrow 0$ uniformly for $x \in \bar{\Omega} \backslash B_{r}$. The $C^{1}$ convergence of $\tilde{u}_{n}(x)$ to $\alpha \nabla_{x} \bar{G}(x, 0)$ follows immediately.

Lemma 2.2. Under the hypotheses of Theorem 0.3, for all $r>0$, there exists some constant $C=C\left(r, \Omega, a, b, A, A_{1}, \alpha\right)$ such that

$$
\max _{\bar{\Omega} \backslash B_{r}} u_{n}-\min _{\bar{\Omega} \backslash B_{r}} u_{n} \leq C
$$

Proof. It follows from Lemma 2.1 and (21) that the oscillation of $u_{n}-\tilde{u}_{n}$ on $\partial \Omega$ is bounded. Since $u_{n}-\tilde{u}_{n}$ is a harmonic function, it follows from the maximum principle that the oscillation of $u_{n}-\tilde{u}_{n}$ in $\bar{\Omega}$ is bounded. Lemma 2.2 follows. 
Due to Lemma 2.2, we only need to establish Theorem 0.3 for a special case: $\Omega=B_{1}$ is the unit ball in $\mathbb{R}^{2}$. Without loss of generality, we assume that $V_{n}(0)=8$. Set

$$
\begin{gathered}
\delta_{n}=e^{-u_{n}(0) / 2} \\
\bar{v}_{n}(x)=u_{n}\left(\delta_{n} x\right)+2 \log \delta_{n}, \quad \text { for }|x| \leq 1 / \delta_{n} \\
\bar{w}_{n}(x)=\bar{v}_{n}(x)+2 \log |x|, \quad \text { for }|x| \leq 1 / \delta_{n} .
\end{gathered}
$$

It is clear that $\bar{v}_{n}$ satisfies

$$
\left\{\begin{aligned}
-\Delta \bar{v}_{n}(x) & =V_{n}\left(\delta_{n} x\right) e^{\bar{v}_{n}(x)} & & \text { for }|x| \leq 1 / \delta_{n} \\
\bar{v}_{n}(x) \leq \bar{v}_{n}(0) & =0 & & \text { for }|x| \leq 1 / \delta_{n}
\end{aligned}\right.
$$

Arguing as in Sect. 1,

$$
\bar{v}_{n} \rightarrow \bar{v} \quad \text { in } C_{l o c}^{2}\left(\mathbb{R}^{2}\right)
$$

and therefore

$$
\bar{w}_{n}-\bar{w} \rightarrow 0 \quad \text { in } C_{l o c}^{2}\left(\mathbb{R}^{2}\right)
$$

where

$$
\begin{aligned}
& \bar{v}(x)=\log \left\{\frac{1}{\left(1+|x|^{2}\right)^{2}}\right\}, \\
& \bar{w}(x)=\log \left\{\frac{|x|^{2}}{\left(1+|x|^{2}\right)^{2}}\right\} .
\end{aligned}
$$

For convenience, we work in cylindrical coordinates $(t, \theta)$ with

$$
\left\{\begin{array}{l}
x_{1}=e^{t} \cos \theta \\
x_{2}=e^{t} \sin \theta
\end{array}\right.
$$

It is easy to check that the transformation given by (26): $\left(x_{1}, x_{2}\right) \rightarrow(t, \cos \theta, \sin \theta)$ is a conformal transformation of $\mathbb{R}^{2} \backslash\{0\}$ to the cylinder $\mathbb{R} \times \mathbb{S}^{1}=\{(t, \cos \theta, \sin \theta)\}$.

Set, for $t<0$ and $\theta \in[0,2 \pi]$,

$$
\tilde{w}_{n}(t, \theta)=u_{n}\left(e^{t} \cos \theta, e^{t} \sin \theta\right)+2 t,
$$

and

$$
\tilde{w}(s)=\log \left\{\frac{e^{2 s}}{\left(1+e^{2 s}\right)^{2}}\right\}=2 s-2 \log \left(1+e^{2 s}\right) .
$$

Under transformation (26),

$$
\bar{w}_{n}(x)=\tilde{w}_{n}\left(t+\log \delta_{n}, \theta\right), \quad \bar{w}(x)=\tilde{w}(t) .
$$

We derive from (25) that in the new variables,

$$
\lim _{n \rightarrow \infty}\left\|\tilde{w}_{n}\left(s+\log \delta_{n}, \theta\right)-\tilde{w}(s)\right\|_{L^{\infty}(s \leq \alpha, \theta \in[0,2 \pi])}=0, \quad \forall \alpha \in \mathbb{R} .
$$

Clearly, under the above conformal transformation of $\mathbb{R}^{2} \backslash\{0\}$ to $\mathbb{R} \times \mathbb{S}^{1}$, the equation of $u_{n}$ is transformed to the following equation on the half cylinder $\mathbb{R}_{-} \times \mathbb{S}^{1}$ : 


$$
-\left(\frac{\partial^{2}}{\partial t^{2}}+\frac{\partial^{2}}{\partial \theta^{2}}\right) \tilde{w}_{n}=\tilde{V}_{n}(t, \theta) e^{\tilde{w}_{n}} \quad \text { in } Q
$$

where

$$
Q=\{(t, \theta): t \leq 0 \text { and } 0 \leq \theta \leq 2 \pi\}
$$

and

$$
\tilde{V}_{n}(t, \theta)=V_{n}\left(e^{t} \cos \theta, e^{t} \sin \theta\right) .
$$

Note that $\tilde{w}$ achieves its maximum at $s=0, \tilde{w}^{\prime}(s)>0$ for $s<0$, and $\tilde{w}(-s)=\tilde{w}(s)$ for all $s$.

Let us first describe the ideas of the proof. For some $R_{n} \rightarrow \infty$, estimate (22) inside the shrinking balls $|x| \leq R_{n} \delta_{n}$ follows from the usual blow up argument. What we need to estimate is in the region $R_{n} \delta_{n} \leq|x| \leq 1$. We work on $\mathbb{R}_{-} \times \mathbb{S}^{1}$, the left half cylinder. It is not difficult to see that the desired estimate (22) in the region $C \delta_{n} \leq|x| \leq 1$ is equivalent to

$$
\left|\tilde{w}_{n}(t, \theta)-\tilde{w}\left(t-\log \delta_{n}\right)\right| \leq C, \quad \forall \log \delta_{n}+C \leq t \leq 0 \text { and } \forall \theta
$$

Here and in the following, $C$ denotes various constants independent of $n$.

The blow up argument gives a precise estimate to $\tilde{w}_{n}(t, \theta)-\tilde{w}\left(t-\log \delta_{n}\right)$ for $t \leq \log \delta_{n}+C$. Since $\tilde{w}\left(t-\log \delta_{n}\right)$ is symmetric with respect to $t=\log \delta_{n}$, estimate $(28)$ is then, in view of (27), equivalent to

$$
\left|\tilde{w}_{n}(t, \theta)-\tilde{w}_{n}\left(2 \log \delta_{n}-t, \theta\right)\right| \leq C, \forall \log \delta_{n}+C \leq t \leq 0 \text { and } \forall \theta .
$$

To establish (29) we will introduce two functions, $\hat{w}_{n}$ and $w_{n}^{*}$, which differ from $\tilde{w}_{n}$ by some uniformly bounded functions. The function $\hat{w}_{n}$ will be chosen so that the method of moving planes can be applied to $\hat{w}_{n}$ from the left to obtain

$$
\hat{w}_{n}(t, \theta) \geq \hat{w}_{n}\left(2 \lambda_{n}-t, \theta\right), \quad \forall \lambda_{n} \leq t \leq 0 \text { and } \forall \theta
$$

where $\lambda_{n}$ is some number smaller than $\log \delta_{n}+2$. On the other hand, $w_{n}^{*}$ will be chosen so that the method of moving planes can be applied to $w_{n}^{*}$ from the right to obtain

$$
w_{n}^{*}(t, \theta) \leq w_{n}^{*}\left(2 \lambda_{n}^{*}-t, \theta\right), \quad \forall \lambda_{n}^{*} \leq t \leq 0 \text { and } \forall \theta
$$

where $\lambda_{n}^{*}$ is some number larger than $\log \delta_{n}-C$. We emphasize that in order to apply the moving plane method to $w_{n}^{*}$ from the right we need (30) and Lemma 2.1-2.2 so that the plane moving process can get started. These estimates are also needed to ensure that

$$
\left|\lambda_{n}-\log \delta_{n}\right|+\left|\lambda_{n}^{*}-\log \delta_{n}\right| \leq C
$$

The desired estimate (29) follows from (30), (31), (32) and (27).

We first introduce

$$
\hat{w}_{n}(t, \theta)=\tilde{w}_{n}(t, \theta)-\frac{A}{a} e^{t} \quad \text { in } Q .
$$


Clearly $\hat{w}_{n}$ satisfies

$$
-\left(\frac{\partial^{2}}{\partial t^{2}}+\frac{\partial^{2}}{\partial \theta^{2}}\right) \hat{w}_{n}=\hat{V}_{n} e^{\hat{w}_{n}}+\frac{A}{a} e^{t}
$$

where

$$
\hat{V}_{n}(t, \theta)=\tilde{V}_{n}(t, \theta) e^{A e^{t} / a}
$$

It is easy to see that

$$
\frac{\partial}{\partial t}\left\{\hat{V}_{n}(t, \theta) e^{\xi}+\frac{A}{a} e^{t}\right\} \geq 0 \quad \forall(t, \theta) \in Q, \forall \xi \in \mathbb{R} .
$$

We recall some estimates obtained for $\hat{w}_{n}$ in [9] by the method of moving planes. For $\lambda<0$ and $\lambda \leq t<0$, we set $t^{\lambda}=2 \lambda-t$ and

$$
\hat{w}_{n}^{\lambda}(t, \theta)=\hat{w}_{n}\left(t^{\lambda}, \theta\right) .
$$

Clearly $\hat{w}_{n}^{\lambda}$ satisfies

$$
-\left(\frac{\partial^{2}}{\partial t^{2}}+\frac{\partial^{2}}{\partial \theta^{2}}\right) \hat{w}_{n}^{\lambda}=\hat{V}_{n}^{\lambda} e^{\hat{w}_{n}^{\lambda}}+\frac{A}{a} e^{t^{\lambda}},
$$

where $\hat{V}_{n}^{\lambda}=\hat{V}_{n}\left(t^{\lambda}, \theta\right)$.

It is easy to see that $\hat{w}_{n}(t, \theta)$ behaves like $2 t$ for $t$ very negative and therefore for $\lambda$ very negative (depending on $n$ ), we have

$$
\hat{w}_{n}^{\lambda}(t, \theta)-\hat{w}_{n}(t, \theta)<0 \quad \text { for } \lambda<t \leq 0,0 \leq \theta \leq 2 \pi .
$$

Define

$\lambda_{n}=\sup \left\{\mu<0: \hat{w}_{n}^{\lambda}(t, \theta)-\hat{w}_{n}(t, \theta)<0\right.$ for all $\left.\lambda<\mu, \lambda<t \leq 0,0 \leq \theta \leq 2 \pi\right\}$.

For every fixed $\alpha \in \mathbb{R}$, we know that $\tilde{w}_{n}(t, \theta)$ approximates $\tilde{w}\left(t-\log \delta_{n}, \theta\right)$ very well in $t \leq \log \delta_{n}+\alpha$. Therefore (see [9] for details)

$$
\lambda_{n} \leq \log \delta_{n}+2 \text {. }
$$

Using the fact

$$
\hat{w}_{n}^{\lambda}(t, \theta)-\hat{w}_{n}(t, \theta)<0 \quad \forall \lambda<t<0, \lambda<\lambda_{n}, 0 \leq \theta \leq 2 \pi,
$$

it is not difficult to see from (34), (33), (35) and the mean value theorem that

$-\left(\frac{\partial^{2}}{\partial t^{2}}+\frac{\partial^{2}}{\partial \theta^{2}}\right)\left(\hat{w}_{n}^{\lambda}(t, \theta)-\hat{w}_{n}(t, \theta)\right) \leq 0 \quad$ for $\lambda \leq t \leq 0, \lambda \leq \lambda_{n}$ and $0 \leq \theta \leq 2 \pi$.

Since the plane moving process stops at $\lambda_{n}$, we derive, using the Hopf lemma and the strong maximum principle, that

$$
\min _{0 \leq \theta \leq 2 \pi}\left\{\hat{w}_{n}(0, \theta)-\hat{w}_{n}\left(2 \lambda_{n}, \theta\right)\right\}=0
$$

Next, we introduce

$$
w_{n}^{*}(t, \theta)=\tilde{w}_{n}(t, \theta)+\frac{A}{a} e^{t} \quad \text { in } Q .
$$


Clearly

$$
-\left(\frac{\partial^{2}}{\partial t^{2}}+\frac{\partial^{2}}{\partial \theta^{2}}\right) w_{n}^{*}=V_{n}^{*} e^{w_{n}^{*}}-\frac{A}{a} e^{t}
$$

where $V_{n}^{*}(t, \theta)=\tilde{V}_{n}(t, \theta) e^{-A e^{t} / a}$.

It is easy to see that

$$
\frac{\partial}{\partial t}\left\{V_{n}^{*}(t, \theta) e^{\xi}-\frac{A}{a} e^{t}\right\}<0 \quad \forall(t, \theta) \in Q, \forall \xi \in \mathbb{R} .
$$

We will apply the method of moving planes to $w_{n}^{*}$, but from the opposite direction. For $\lambda<0$ and $2 \lambda \leq t \leq \lambda$, we set

$$
w_{n}^{* \lambda}(t, \theta)=w_{n}^{*}\left(t^{\lambda}, \theta\right)
$$

where, as before, $t^{\lambda}=2 \lambda-t$.

Clearly

$$
-\left(\frac{\partial^{2}}{\partial t^{2}}+\frac{\partial^{2}}{\partial \theta^{2}}\right) w_{n}^{* \lambda}=V_{n}^{* \lambda}(t, \theta) e^{w_{n}^{* \lambda}}-\frac{A}{a} e^{t^{\lambda}},
$$

where $V_{n}^{* \lambda}(t, \theta)=V_{n}^{*}\left(t^{\lambda}, \theta\right)$.

In order to get started with the plane moving process, appropriate estimates are needed for $w_{n}^{*}$. For that purpose, we first use the harmonicity of $u_{n}-\tilde{u}_{n}$ in $B_{1}$, the boundedness of the oscillation of $u_{n}-\tilde{u}_{n}$ in $B_{1}$, and standard elliptic estimates to obtain

$$
\left|\nabla\left(u_{n}-\tilde{u}_{n}\right)\right| \leq C \quad \text { in } B_{1 / 2} .
$$

Taking $-\Lambda_{1}>-\Lambda_{2}>>1$, we derive from (41) and Lemma 2.1, for large $n$ (depending on $\Lambda_{1}$ and $\Lambda_{2}$ ), that

$$
\frac{\partial u_{n}}{\partial t}(t, \theta) \leq-\frac{\alpha}{2 \pi}+1, \quad \forall \Lambda_{1} \leq t \leq \Lambda_{2}, 0 \leq \theta \leq 2 \pi
$$

Notice that $\alpha \geq 8 \pi$, we have

$$
\frac{\partial \tilde{w}_{n}}{\partial t}(t, \theta)=\frac{\partial u_{n}}{\partial t}(t, \theta)+2 \leq-1, \quad \forall \Lambda_{1} \leq t \leq \Lambda_{2}, 0 \leq \theta \leq 2 \pi .
$$

Consequently,

$$
\frac{\partial w_{n}^{*}}{\partial t}(t, \theta) \leq-1 / 2, \quad \forall \Lambda_{1} \leq t \leq \Lambda_{2}, 0 \leq t \leq \theta .
$$

Fix $\Lambda_{2}$ first. It follows from (37), (27), (36) and Lemma 2.2 that

$$
w_{n}^{*}(t, \theta) \leq \tilde{w}\left(2 \lambda_{n}-\log \delta_{n}\right)+C\left(\Lambda_{2}\right) \leq 2\left(2 \lambda_{n}-\log \delta_{n}\right)+C\left(\Lambda_{2}\right)
$$

for $\Lambda_{2} \leq t \leq 0,0 \leq \theta \leq 2 \pi$. Therefore, for all $\Lambda_{0}<\Lambda_{2}, 2 \Lambda_{0} \leq t \leq 2 \Lambda_{0}-\Lambda_{2}$, we have

$$
w_{n}^{* \Lambda_{0}}(t, \theta)=w_{n}^{*}\left(t^{\Lambda_{0}}, \theta\right) \leq 2\left(2 \lambda_{n}-\log \delta_{n}\right)+C\left(\Lambda_{2}\right) .
$$


Using the definition of $\lambda_{n}$, we have

$$
w_{n}^{*}(t, \theta) \geq \hat{w}_{n}(t, \theta)-C \geq \hat{w}_{n}^{\lambda_{n}}(t, \theta)-C \quad \forall \lambda_{n} \leq t \leq 0,0 \leq \theta \leq 2 \pi,
$$

where $C$ is some constant independent of $n, \Lambda_{2}, \Lambda_{1}$ and $\Lambda_{0}$. Namely, for all $\lambda_{n} \leq t \leq$ $0,0 \leq \theta \leq 2 \pi$ we have

$$
w_{n}^{*}(t, \theta) \geq \hat{w}_{n}\left(2 \lambda_{n}-t, \theta\right)-C .
$$

Therefore for all $\lambda_{n} \leq t \leq \Lambda_{0}, 0 \leq \theta \leq 2 \pi$, we have, in view of (36) and (27),

$$
\begin{aligned}
w_{n}^{*}(t, \theta) & \geq \hat{w}_{n}\left(2 \lambda_{n}-t, \theta\right)-C \\
& \geq \tilde{w}\left(2 \lambda_{n}-\log \delta_{n}-t, \theta\right)-C \\
& \geq 2\left(2 \lambda_{n}-\log \delta_{n}-t\right)-C .
\end{aligned}
$$

We see from (43) and (44) that there exists some $\bar{\Lambda}_{0}<\Lambda_{2}$ such that for all $\Lambda_{0}<\bar{\Lambda}_{0}$, and all $\lambda_{n} \leq 2 \Lambda_{0} \leq t \leq 2 \Lambda_{0}-\Lambda_{2}$ and $0 \leq \theta \leq 2 \pi$, we have

$$
w_{n}^{* \Lambda_{0}}(t, \theta)<w_{n}^{*}(t, \theta) .
$$

Fix one such $\Lambda_{0}<\bar{\Lambda}_{0}$.

Using (42) with $\Lambda_{1}=2 \Lambda_{0}$, we have, for $n$ large,

$$
w_{n}^{* \Lambda_{0}}(t, \theta)<w_{n}^{*}(t, \theta), \quad \forall 2 \Lambda_{0}-\Lambda_{2} \leq t<\Lambda_{0}, 0 \leq \theta \leq 2 \pi .
$$

Define

$\lambda_{n}^{*}=\inf \left\{\mu \leq \Lambda_{0}: w_{n}^{* \lambda}(t, \theta)-w_{n}^{*}(t, \theta)<0 \forall \mu \leq \lambda \leq \Lambda_{0}, 2 \lambda \leq t<\lambda, 0 \leq \theta \leq 2 \pi\right\}$.

Due to (45) and (46), $\lambda_{n}^{*}$ is well defined for large $n$. It is easy to see from (27), for large $n$, that

$$
\lambda_{n}^{*} \geq \log \delta_{n}-2
$$

Using the fact

$$
w_{n}^{* \lambda}(t, \theta)-w_{n}^{*}(t, \theta)<0, \quad \forall 2 \lambda<t<\lambda, \lambda_{n}^{*} \leq \lambda \leq \Lambda_{0}, 0 \leq \theta \leq 2 \pi,
$$

we derive from (38), (40), (39) and the mean value theorem that

$$
\begin{gathered}
-\left(\frac{\partial^{2}}{\partial t^{2}}+\frac{\partial^{2}}{\partial \theta^{2}}\right)\left(w_{n}^{* \lambda}(t, \theta)-w_{n}^{*}(t, \theta)\right) \leq 0 \\
\forall 2 \lambda<t<\lambda, \lambda_{n}^{*} \leq \lambda \leq \Lambda_{0}, 0 \leq \theta \leq 2 \pi .
\end{gathered}
$$

Since the plane moving process stops at $\lambda_{n}^{*}$, we have, by using the strong maximum principle and the Hopf lemma, that

$$
\max _{0 \leq \theta \leq 2 \pi}\left\{w_{n}^{* \lambda_{n}^{*}}\left(2 \lambda_{n}^{*}, \theta\right)-w_{n}^{*}\left(2 \lambda_{n}^{*}, \theta\right)\right\}=0 .
$$

Namely,

$$
\max _{0 \leq \theta \leq 2 \pi}\left\{w_{n}^{*}(0, \theta)-w_{n}^{*}\left(2 \lambda_{n}^{*}, \theta\right)\right\}=0
$$


It follows from the definition of $w_{n}^{*}$ and (21) that

$$
\min _{\partial B_{1}} u_{n}-C \leq w_{n}^{*}(0, \theta) \leq \min _{\partial B_{1}} u_{n}+C \quad \forall 0 \leq \theta \leq 2 \pi .
$$

Using (27) and the definition of $\lambda_{n}$, we also know that

$$
\begin{aligned}
\min _{0 \leq \theta \leq 2 \pi} w_{n}^{*}\left(2 \lambda_{n}^{*}, \theta\right) & \geq \min _{0 \leq \theta \leq 2 \pi} \hat{w}_{n}\left(2 \lambda_{n}^{*}, \theta\right)-C \\
& \geq\left\{\begin{array}{r}
\min _{0 \leq \theta \leq 2 \pi} \hat{w}_{n}\left(2 \lambda_{n}-2 \lambda_{n}^{*}\right)-C \text { if } 2 \lambda_{n}^{*} \geq \lambda_{n} \\
\min _{0 \leq \theta \leq 2 \pi} \hat{w}_{n}\left(2 \lambda_{n}^{*}\right)-C \text { if } 2 \lambda_{n}^{*}<\lambda_{n}
\end{array}\right. \\
& \geq\left\{\begin{array}{r}
2\left(2 \lambda_{n}-2 \lambda_{n}^{*}-\log \delta_{n}\right)-C \text { if } 2 \lambda_{n}^{*} \geq \lambda_{n} \\
2\left(2 \lambda_{n}^{*}-\log \delta_{n}\right)-C \text { if } 2 \lambda_{n}^{*}<\lambda_{n}
\end{array}\right.
\end{aligned}
$$

Combining (48), (49) and (50), we have

$$
\max _{\partial B_{1}} u_{n} \geq\left\{\begin{array}{r}
2\left(2 \lambda_{n}-2 \lambda_{n}^{*}-\log \delta_{n}\right)-C \text { if } 2 \lambda_{n}^{*} \geq \lambda_{n} \\
2\left(2 \lambda_{n}^{*}-\log \delta_{n}\right)-C \text { if } 2 \lambda_{n}^{*}<\lambda_{n}
\end{array} .\right.
$$

On the other hand, we know from (37) and (27) that

$$
\min _{\partial B_{1}} u_{n} \leq 2\left(2 \lambda_{n}-\log \delta_{n}\right)+C .
$$

It follows from (21), (51), (52) that either

$$
-\lambda_{n}^{*} \leq C,
$$

or

$$
\lambda_{n}^{*} \leq \lambda_{n}+C
$$

We rule out (53) as follows. Suppose (53) happens, then, since $\lambda_{n}^{*}<\bar{\Lambda}_{0}$, we derive from (45), for all $2 \lambda_{n}^{*} \leq t \leq 2 \lambda_{n}^{*}-\Lambda_{2}$ and $0 \leq \theta \leq 2 \pi$, that

$$
w_{n}^{* \lambda_{n}^{*}}(t, \theta)<w_{n}^{*}(t, \theta) .
$$

Now, in view of (42), we have, for $n$ large, $2 \lambda_{n}^{*}-\Lambda_{2} \leq t \leq \Lambda_{2}$, and $0 \leq \theta \leq 2 \pi$, that

$$
\frac{\partial w_{n}^{*}}{\partial t}(t, \theta) \leq-1 / 2<0 .
$$

These imply, for some $\epsilon>0$ and $\lambda \in\left[\lambda_{n}^{*}-\epsilon, \lambda_{n}^{*}\right]$, that

$$
w_{n}^{* \lambda}(t, \theta)<w_{n}^{*}(t, \theta)
$$

for all $2 \lambda \leq t<\lambda$ and $0 \leq \theta \leq 2 \pi$. This violates the definition of $\lambda_{n}^{*}$, so (53) can not happen. Therefore we always have (54) and, in view of (47) and (36), that

$$
\left|\lambda_{n}-\log \delta_{n}\right|+\left|\lambda_{n}^{*}-\log \delta_{n}\right| \leq C .
$$


Recall that

$$
w_{n}^{* \lambda_{n}^{*}}(t, \theta) \leq w_{n}^{*}(t, \theta), \quad \forall 2 \lambda_{n}^{*} \leq t \leq \lambda_{n}^{*}, 0 \leq \theta \leq 2 \pi,
$$

and

$$
\hat{w}_{n}^{\lambda_{n}}(t, \theta) \leq \hat{w}_{n}(t, \theta), \quad \forall \lambda_{n} \leq t \leq 0,0 \leq \theta \leq 2 \pi .
$$

Namely,

$$
w_{n}^{*}(t, \theta) \leq w_{n}^{*}\left(2 \lambda_{n}^{*}-t, \theta\right), \quad \forall \lambda_{n}^{*} \leq t \leq 0,0 \leq \theta \leq 2 \pi,
$$

and

$$
\hat{w}_{n}(t, \theta) \geq \hat{w}_{n}\left(2 \lambda_{n}-t, \theta\right), \quad \forall \lambda_{n} \leq t \leq 0,0 \leq \theta \leq 2 \pi .
$$

Since

$$
\left|\hat{w}_{n}(t, \theta)-\tilde{w}_{n}(t, \theta)\right|+\left|w_{n}^{*}(t, \theta)-\tilde{w}_{n}(t, \theta)\right| \leq C,
$$

for all $t \leq 0$ and $\theta$, we have

$$
\left\{\begin{array}{l}
\tilde{w}_{n}(t, \theta) \leq \tilde{w}_{n}\left(2 \lambda_{n}^{*}-t, \theta\right)+C, \forall \lambda_{n}^{*} \leq t \leq 0,0 \leq \theta \leq 2 \pi \\
\tilde{w}_{n}(t, \theta) \geq \tilde{w}_{n}\left(2 \lambda_{n}-t, \theta\right)-C, \forall \lambda_{n} \leq t \leq 0,0 \leq \theta \leq 2 \pi
\end{array}\right.
$$

Due to (55), we have

$$
2 \lambda_{n}^{*}-t \leq \log \delta_{n}+C \quad \forall \lambda_{n}^{*} \leq t \leq 0,
$$

and

$$
2 \lambda_{n}-t \leq \log \delta_{n}+C \quad \forall \lambda_{n} \leq t \leq 0 .
$$

So we can use (27) to estimate the right hand sides of (56) and obtain, using again (55), that

$2\left(\log \delta_{n}-t\right)-C \leq \tilde{w}_{n}(t, \theta) \leq 2\left(\log \delta_{n}-t\right)+C, \quad \forall \log \delta_{n} \leq t \leq 0, \forall \theta$.

In terms of $u_{n}$, this means

$$
\left|u_{n}(x)+u_{n}(0)+4 \log \right| x|| \leq C, \quad \forall \delta_{n} \leq|x| \leq 1
$$

The standard blow up argument (see (24)) yields, for some $R_{n} \rightarrow \infty$,

$$
\max _{|x| \leq R_{n} \delta_{n}}\left|u_{n}(x)-\log \frac{\delta_{n}^{-2}}{\left(1+\delta_{n}^{-2}|x|^{2}\right)^{2}}\right| \rightarrow 0 \quad \text { as } n \rightarrow \infty .
$$

On the other hand, (57) is equivalent to

$$
\left|u_{n}(x)-\log \frac{\delta_{n}^{-2}}{\left(1+\delta_{n}^{-2}|x|^{2}\right)^{2}}\right| \leq C, \forall \delta_{n} \leq|x| \leq 1 .
$$

Theorem 0.3 follows from the above two estimates. 


\section{Proof of Theorem 0.2}

In this section we establish Theorem 0.2 by using Theorem 0.3 .

Proof of Theorem 0.2. We know from Theorem 0.1 that $\bar{\lambda} \in[8 \pi, \infty)$. For any point $\bar{y} \in M$, let $x=\left(x_{1}, x_{2}\right)$ be some isothermal coordinate system centered at $\bar{y}$. The metric $g$ takes the form $e^{\varphi}\left(d x_{1}^{2}+d x_{2}^{2}\right)$ in $B_{r}(0):=\left\{x \mid x_{1}^{2}+x_{2}^{2}<r\right\}$ with $\varphi(0)=0$. Then $\xi_{n}$ satisfies

$$
-\Delta \xi_{n}=\lambda_{n} e^{\varphi}\left(V_{n} e^{\xi_{n}}-W_{n}\right), \quad \text { in } x_{1}^{2}+x_{2}^{2}<r,
$$

where $\Delta=\partial_{x_{1} x_{1}}+\partial_{x_{2} x_{2}}$. Define $\zeta_{n}$ by

$$
\begin{cases}-\Delta \zeta_{n}=\lambda_{n} e^{\varphi} W_{n}+\Delta \varphi, & \text { in } B_{r}(0), \\ \zeta_{n}=0, & \text { on } \partial B_{r}(0),\end{cases}
$$

and set $\eta_{n}=\xi_{n}+\zeta_{n}+\varphi$. Then $\eta_{n}$ satisfies

$$
-\Delta \eta_{n}=\lambda_{n} e^{-\zeta_{n}} V_{n} e^{\eta_{n}}, \text { in } B_{r}(0) .
$$

It is clear that $\left\{\zeta_{n}\right\}$ is uniformly bounded in $\bar{B}_{r}(0)$. We see from (7) that $\left\{\int_{M} e^{\xi_{n}} d v_{g}\right\}$ is bounded from above, so $\lambda_{n} \int_{B_{r}(0)} e^{-\zeta_{n}} V_{n} e^{\eta_{n}} \leq C$. Therefore it follows from Theorem 3 of [8] that, after passing to a subsequence, there are only three possibilities:

(i) $\left\{\eta_{n}\right\}$ uniformly converges in $C^{2}\left(\bar{B}_{r / 2}(0)\right)$,

(ii) $\left\{\eta_{n}\right\}$ tends to $-\infty$ uniformly on $\bar{B}_{r / 2}(0)$,

(iii) There exist finitely many blowup points $\left\{x^{(1)}, \cdots, x^{(l)}\right\}$ of $\left\{\eta_{n}\right\}$ such that $\left\{\eta_{n}\right\}$ tends to $-\infty$ uniformly on compact subsets of $\bar{B}_{r / 2}(0) \backslash\left\{x^{(1)}, \cdots, x^{(l)}\right\}$.

Clearly, in view of the boundedness of $\left\{\zeta_{n}\right\}$, there are only the above three possibilities for $\left\{\xi_{n}\right\}$ as well. Since $M$ is connected, we know that, after passing to a subsequence, there are only three possibilities for $\left\{\xi_{n}\right\}$ on $M$ :

$1^{\circ}\left\{\xi_{n}\right\}$ uniformly converges in $C^{2}(M)$,

$2^{\circ}\left\{\xi_{n}\right\}$ tends to $-\infty$ uniformly on $M$,

$3^{\circ}$ There exist finitely many blowup points $\left\{\bar{x}^{(1)}, \cdots, \bar{x}^{(m)}\right\}$ of $\left\{\xi_{n}\right\}$ such that $\left\{\xi_{n}\right\}$ tends to $-\infty$ uniformly on compact subsets of $M \backslash\left\{\bar{x}^{(1)}, \cdots, \bar{x}^{(m)}\right\}$.

Since we know from (7) that $\left\{\int_{M} e^{\xi_{n}} d v_{g}\right\}$ has a positive lower bound, so $2^{\circ}$ can not occur. $1^{\circ}$ can not occur either because of (9). We are left with $3^{\circ}$. Applying the result in [32], we know that $\lambda_{n} V_{n} e^{\xi_{n}} \rightarrow \sum_{l=1}^{m} 8 \pi N_{l} \delta_{\bar{x}^{(l)}}$ for some positive integers $N_{l}$. Consequently, in view of (7), $\bar{\lambda}=8 \pi \sum_{l=1}^{m} N_{l}$. We then derive from (8) that, in $C_{l o c}^{0}\left(M \backslash\left\{\bar{x}^{(1)}, \cdots, \bar{x}^{(m)}\right\}\right)$,

$$
\xi_{n}-\bar{\xi}_{n} \rightarrow 8 \pi \sum_{l=1}^{m} N_{l} G\left(\cdot, \bar{x}^{(l)}\right)-\bar{\lambda} \int_{M} W(y) G(\cdot, y) d v_{g}(y)
$$

Due to (58), $\left\{\xi_{n}\right\}$ has bounded oscillations in compact subsets of $M \backslash\left\{\bar{x}^{(1)}, \cdots, \bar{x}^{(m)}\right\}$. Let $0<a_{l}<\frac{1}{2} \min _{l^{\prime} \neq l} d\left(\bar{x}^{(l)}, \bar{x}^{\left(l^{\prime}\right)}\right)$ be some small constant, $\bar{x}_{n}^{(l)}$ be a maximum point of $\xi_{n}$ in $\left\{y \in M \mid d\left(y, \bar{x}^{(l)}\right)<a_{l}\right\}$, and $x=\left(x_{1}, x_{2}\right)$ be some isothermal coordinate system centered at $\bar{x}_{n}^{(l)}$. The metric $g$ takes the form $e^{\varphi_{n}}\left(d x_{1}^{2}+d x_{2}^{2}\right)$ in $B_{a_{l}}(0):=\left\{x \mid x_{1}^{2}+x_{2}^{2}<a_{l}\right\}$ with $\varphi_{n}(0)=0$. Define $\zeta_{n}$ and $\eta_{n}$ in $B_{r}(0)$ as at the beginning of this section with $r=a_{l}$, then, by applying Theorem 0.3 to $\eta_{n}$, we have 


$$
\left|\eta_{n}(x)-\log \frac{e^{\eta_{n}(0)}}{\left(1+\frac{\lambda_{n} e^{-\zeta_{n}(0)} V_{n}(0)}{8} e^{\eta_{n}(0)}|x|^{2}\right)^{2}}\right| \leq C, \quad \forall|x| \leq a_{l},
$$

namely,

$$
\left|\xi_{n}(x)-\log \frac{e^{\xi_{n}(0)}}{\left(1+\frac{\lambda_{n} V_{n}(0)}{8} e^{\xi_{n}(0)}|x|^{2}\right)^{2}}\right| \leq C, \quad \forall|x| \leq a_{l} .
$$

It follows easily that

$$
\lambda_{n} V_{n} e^{\xi_{n}} \rightarrow 8 \pi \sum_{l=1}^{m} \delta_{\bar{x}^{(l)}} \text { in the sense of measure. }
$$

In the isothermal coordinate system centered at $\bar{x}_{n}^{(l)}$, we define

$$
v_{n}(x)=\xi_{n}\left(\delta_{n} x\right)+2 \log \delta_{n}, \quad|x|<a_{l} \delta_{n}^{-1},
$$

where $\delta_{n}=e^{-\xi_{n}(0) / 2} \rightarrow 0$. Set

$$
\bar{R}_{n}^{(l)}:=\sup \left\{R>0:\left\|v_{n}-v\right\|_{C^{2}\left(\bar{B}_{2 R}(0)\right)}+\left\|v_{n}-v\right\|_{H^{2}\left(\bar{B}_{2 R}(0)\right)}<e^{-R}\right\},
$$

where

$$
v(x)=\log \left\{\frac{1}{\left(1+\frac{\bar{\lambda} \lim _{n \rightarrow \infty} V_{n}(0)}{8}|x|^{2}\right)^{2}}\right\}, \quad \text { in } \mathbb{R}^{2} .
$$

Arguing by contradiction using the standard blow up argument as in Sect. 1, we can show that $\bar{R}_{n}^{(l)} \rightarrow \infty$ as $n \rightarrow \infty$. Clearly,

$$
\lambda_{n} \int_{d\left(y, \bar{x}_{n}^{(l)}\right)<\bar{R}_{n}^{(l)} e^{-\xi_{n}\left(\bar{x}_{n}^{(l)}\right) / 2}} V_{n} e^{\xi_{n}} \rightarrow 8 \pi,
$$

and $\xi_{n}$, for large $n$, has a unique critical point in $\left\{y \in M \mid d\left(y, \bar{x}_{n}^{(l)}\right)<\bar{R}_{n}^{(l)} e^{-\xi_{n}\left(\bar{x}_{n}^{(l)}\right) / 2}\right\}$ due to the fact that $v$ has a unique nondegenerate critical point at the origin. It is easy to see from (59) that

$$
\int_{\bar{R}_{n}^{(l)} e^{-\xi_{n}\left(\bar{x}_{n}^{(l)}\right) / 2}<d\left(y, \bar{x}_{n}^{(l)}\right)<a_{l}} V_{n} e^{\xi_{n}} \rightarrow 0 .
$$

Consequently, $N_{l}=1$ for all $l$ and $\bar{\lambda}=8 \pi m$. (e) then follows from (58). We easily derive (d) and (c) from (59) and (58).

The above discussion also yields the uniqueness of the maximum point $\bar{x}_{n}^{(l)}$ since

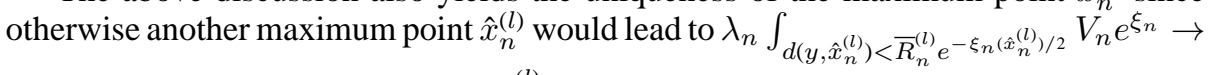
$8 \pi$, and due to the definition of $\bar{R}_{n}^{(l)}$, the two small balls $\left\{y \in M \mid d\left(y, \bar{x}_{n}^{(l)}\right)<\right.$ $\left.\bar{R}_{n}^{(l)} e^{-\xi_{n}\left(\bar{x}_{n}^{(l)}\right) / 2}\right\}$ and $\left\{y \in M \mid d\left(y, \hat{x}_{n}^{(l)}\right)<\bar{R}_{n}^{(l)} e^{-\xi_{n}\left(\hat{x}_{n}^{(l)}\right) / 2}\right\}$ have no intersections. This would violate $N_{l}=1$. Theorem 0.2 is thus established.

In the rest of this section we derive Corollary 0.3 from Theorem 0.2.

Proof of Corollary 0.3. Using (8), we write $\varphi_{n}$ as

$$
\varphi_{n}=\varphi_{n}^{(1)}+\varphi_{n}^{(2)}
$$


with

$$
\varphi_{n}^{(1)}:=\lambda_{n} \int_{M} V_{n}(y) e^{\xi_{n}(y)} G(\cdot, y) d v_{g}(y)-8 \pi \sum_{l=1}^{m} G\left(\cdot, \bar{x}_{n}^{(l)}\right)
$$

and

$$
\varphi_{n}^{(2)}:=8 \pi m \int_{M} W(y) G(\cdot, y) d v_{g}(y)-\lambda_{n} \int_{M} W_{n}(y) G(\cdot, y) d v_{g}(y) .
$$

Since $\lambda_{n} W_{n} \rightarrow 8 \pi m W$ in $C^{\alpha}(M)$ for $0<\alpha<1$, we derive from Schauder estimates that $\varphi_{n}^{(2)} \rightarrow 0$ in $C^{2, \alpha}(M)$, so

$$
\varphi_{n}^{(2)} \sim 0
$$

Without loss of generality, we may assume $d\left(x, \bar{x}_{n}^{(1)}\right)=\min _{1 \leq l \leq m} d\left(x, \bar{x}_{n}^{(l)}\right)$. Write

$$
\varphi_{n}^{(1)}=\varphi_{n}^{(11)}+\varphi_{n}^{(12)}+\varphi_{n}^{(13)}+\varphi_{n}^{(14)}
$$

with

$$
\begin{gathered}
\varphi_{n}^{(11)}=\lambda_{n} \sum_{l=1}^{m} \int_{B}{ }_{\overline{\bar{R}}_{n}^{(l)} \delta_{n}^{(l)}\left(\bar{x}_{n}^{(l)}\right)} V_{n}(y) e^{\xi_{n}(y)}\left[G(\cdot, y)-G\left(\cdot, \bar{x}_{n}^{(l)}\right)\right] d v_{g}(y), \\
\varphi_{n}^{(12)}=\sum_{l=1}^{m}\left(\int_{B} \lambda_{\bar{R}_{n}^{(l)} \delta_{n}^{(l)}}\left(\bar{x}_{n}^{(l)}\right)\right. \\
\left.\varphi_{n}^{(13)}=\lambda_{n} \int_{d(y, x) \leq d\left(x, \bar{x}_{n}^{(1)}\right) / 4} V_{n}(y) e^{\xi_{n}(y)} d v_{g}(y)-8 \pi\right) G\left(\cdot, \bar{x}_{n}^{(l)}\right), \\
\varphi_{n}^{(14)}=\lambda_{n} \int_{d(y, x) \geq d\left(x, \bar{x}_{n}^{(1)}\right) / 4, y \in M \backslash \cup_{l=1}^{m} B}{\sqrt{\bar{R}_{n}^{(l)}} \delta_{n}^{(l)}\left(\bar{x}_{n}^{(l)}\right)}_{n} V_{n}(y) e^{\xi_{n}(y)} G(\cdot, y) d v_{g}(y),
\end{gathered}
$$

For $x \in M \backslash \cup_{l=1}^{m} B_{\bar{R}_{n}^{(l)}} \delta_{n}^{(l)}\left(\bar{x}_{n}^{(l)}\right)$, we derive from (c)-(d) in Theorem 0.2 that

$$
\begin{aligned}
\left|\varphi^{(13)}(x)\right| & \leq C \frac{e^{\xi_{n}\left(\bar{x}_{n}^{(1)}\right)}}{\left(1+e^{\xi_{n}\left(\bar{x}_{n}^{(1)}\right)} d\left(x, \bar{x}_{n}^{(1)}\right)^{2}\right)^{2}} \int_{d(y, x) \leq d\left(x, \bar{x}_{n}^{(1)}\right) / 4}|G(x, y)| d v_{g}(y) \\
& \leq C\left(1+\left|\log d\left(x, \bar{x}_{n}^{(1)}\right)\right|\right) \frac{e^{\xi_{n}\left(\bar{x}_{n}^{(1)}\right)} d\left(x, \bar{x}_{n}^{(1)}\right)^{2}}{\left(1+e^{\xi_{n}\left(\bar{x}_{n}^{(1)}\right)} d\left(x, \bar{x}_{n}^{(1)}\right)^{2}\right)^{2}},
\end{aligned}
$$

which implies, in view of

$$
\left.e^{\xi_{n}\left(\bar{x}_{n}^{(1)}\right)} d\left(x, \bar{x}_{n}^{(1)}\right)^{2} \geq e^{\xi_{n}\left(\bar{x}_{n}^{(1)}\right)}\left(\bar{R}_{n}^{(1)} \delta_{n}^{(1)}\right)\right)^{2}=\left(\bar{R}_{n}^{(1)}\right)^{2} \rightarrow \infty,
$$

that

$$
\varphi^{(13)} \sim_{0} 0
$$

The usual blow up argument as in Sect. 1 yields

$$
\varphi^{(12)} \sim_{0} 0
$$


Apparently, for $x \in M \backslash \cup_{l=1}^{m} B_{\text {overline } R_{n}^{(l)} \delta_{n}^{(l)}}\left(\bar{x}_{n}^{(l)}\right)$,

$$
\begin{aligned}
\left|\varphi^{(11)}(x)\right| & \leq C \sum_{l=1}^{m} \int_{B}{\sqrt{\bar{R}_{n}^{(l)}}\left(l()_{n}^{\left(x_{n}^{l l}\right)}\right.}_{e^{\xi_{n}(y)}}\left|\log \frac{d(x, y)}{d\left(x, \bar{x}_{n}^{(i)}\right)}\right| d v_{g}(y) \\
& \leq C \sum_{l=1}^{m} \log \frac{\bar{R}_{n}^{(l)}+\sqrt{\bar{R}_{n}^{(l)}}}{\bar{R}_{n}^{(l)}-\sqrt{\bar{R}_{n}^{(l)}}} .
\end{aligned}
$$

Consequently,

$$
\varphi^{(11)} \sim_{0} 0
$$

Using (a) and (c), we have

$$
\begin{aligned}
\left|\varphi^{(14)}(x)\right| & \leq C\left(1+\left|\log d\left(x, \bar{x}_{n}^{(1)}\right)\right|\right) \int_{M \backslash \cup_{l=1}^{m} B_{\bar{R}_{n}^{(l)} \delta_{n}^{(l)}}\left(\bar{x}_{n}^{(1)}\right)} e^{\xi_{n}} d v_{g} \\
& =\circ(1)\left(1+\left|\log d\left(x, \bar{x}_{n}^{(l)}\right)\right|\right) .
\end{aligned}
$$

Namely,

$$
\varphi^{(14)} \sim_{0} 0
$$

Combining (61)-(64), we have

$$
\varphi^{(1)} \sim_{0} 0 .
$$

Differentiating $\varphi^{(1)}$ under the integral sign and making estimates as above, we can easily show (details are left to readers) that

$$
\varphi^{(1)} \sim_{1} 0 \quad \text { and } \quad \varphi^{(1)} \sim_{2} 0 .
$$

Therefore

$$
\varphi^{(1)} \sim 0 .
$$

Corollary 0.3 follows from (60) and (65).

\section{Appendix}

For readers' convenience, we provide a proof of the following well known fact.

Lemma 4.1. There is no $C^{2}$ solution to

$$
\left\{\begin{array}{l}
\Delta v=e^{v}, \quad \text { in } \mathbb{R}^{2}, \\
\int_{\mathbb{R}^{2}} e^{v}<\infty .
\end{array}\right.
$$

Proof. Suppose the contrary, $v$ is a $C^{2}$ solution. Set

$$
\bar{v}(r)=\frac{1}{2 \pi r} \int_{\partial B_{r}} v
$$

for $r>0$. We derive from Jensen's inequality that

$$
\frac{1}{2 \pi r} \int_{\partial B_{r}} e^{v} \geq e^{\bar{v}(r)} .
$$


It follows that $\bar{v}$ satisfies

$$
\Delta \bar{v} \geq e^{\bar{v}(r)}, \quad \text { in } \mathbb{R}^{2},
$$

namely,

$$
\frac{1}{r}\left(r \bar{v}^{\prime}(r)\right)^{\prime} \geq e^{\bar{v}(r)}
$$

We derive from the above that

$$
r \bar{v}^{\prime}(r) \geq \int_{0}^{r} s e^{\bar{v}(s)} \geq 0 \quad \text { for all } r \geq 0 .
$$

Consequently,

$$
r \bar{v}^{\prime}(r) \geq \int_{0}^{r} s e^{\bar{v}(0)}=e^{\bar{v}(0)} \frac{r^{2}}{2} \quad \text { for all } r \geq 0 .
$$

In turn we have

$$
\bar{v}(r) \geq \bar{v}(0)+e^{\bar{v}(0)} \frac{r^{2}}{4} \quad \text { for all } r \geq 0 .
$$

It follows from (66) and (67) that

$$
\int_{\mathbb{R}^{2}} e^{v}=\infty
$$

Contradiction.

\section{References}

1. Alexandrov, A.D.: Uniqueness theorems for surfaces in the large I-V. Vestnik Leningrad Univ. 11 \#19, 5-17 (1956); 12 \#7, 15-44 (1957); 13 \#7, 14-26 (1958); 13 \#13, 27-34 (1958); 13 \#19, 5-8 (1958); English transl. in Am. Math. Soc. Transl. 21, 341-354, 354-388, 389-403, 403-411, 412-416 (1962)

2. Aubin, T.: Nonlinear Analysis on Manifolds. Monge-Ampère Equations. New York-Berlin: SpringerVerlag, 1982

3. Berestycki, H., Caffarelli, L. and Nirenberg, L.: Symmetry for elliptic equations in a half space, Boundary value problems for partial differential equations and applications. RMA Res. Notes Appl. Math., 29, Paris: Masson, 1993, pp. 27-42

4. Berestycki, H., Caffarelli, L. and Nirenberg, L.: Further qualitative properties for elliptic equations in unbounded domains. Annali Sc. Norm. Sup. Pisa Cl. Sci. 4, 1 (1998)

5. Berestycki, H. and Nirenberg, L.: Monotonicity, symmetry and antisymmetry of solutions of semilinear elliptic equations. J. Geom. Phys. 5, 237-275 (1988)

6. Berestycki, H. and Nirenberg, L.: Some qualitative properties of solutions of semilinear elliptic equations in cylindrical domains. Analysis, et cetera, Boston, MA: Academic Press, 1990, pp. 115-164

7. Berestycki, H. and Nirenberg, L.: On the method of moving planes and the sliding method. Bol. Soc. Bras. Mat. 22, 1-37 (1991)

8. Brezis, H. and Merle, F.: Uniform estimates and blow-up behavior for solutions of $-\Delta u=V(x) e^{u}$ in two dimension. Commun. Partial Differential Equation 16, 1223-1253 (1991)

9. Brezis, H., Li, Y.Y. and Shafrir, I.: A sup + inf inequality for some nonlinear elliptic equations involving exponential nonlinearities. J. Funct. Anal. 115, 344-358 (1993)

10. Caffarelli, L., Gidas, B. and Spruck, J.: Asymptotic symmetry and local behavior of semilinear elliptic equations with critical Sobolev growth. Commun. Pure Appl. Math. 42, 271-297 (1989)

11. Caffarelli, L. and Yang, Y.: Vortex condensation in the Chern-Simons Higgs model: an existence theorem. Commun. Math. Phys. 168, 321-336 (1995)

12. Caglioti, E., Lions, P.L. and Marchioro, C.: A special class of stationary flows for two-dimensional Euler equations: A statistical mechanics description. Commun. Math. Phys. 143, 501-525 (1992) 
13. Caglioti, E., Lions, P.L., Marchioro, C. and Pulvirenti, M.: A special class of stationary flows for twodimensional Euler equations: A statistical mechanics description, part II. Commun. Math. Phys. 174, 229-260 (1995)

14. Carleson, L. and Chang, S.Y.: On the existence of an extremal function for an inequality of Moser. Bull. Sci. Math. 110, 113-127 (1986)

15. Chanillo, S. and Kiessling, M.K.H.: Conformally invariant systems of nonlinear PDE of Liouville type. Geom. Funct. Anal. 5, 924-947 (1995)

16. Chang, S.Y. and Yang, P.: A perturbation result in prescribing scalar curvature on $\mathbb{S}^{n}$. Duke Math. J. 64, 27-69 (1991)

17. Chen, C.C. and Lin, C.S.: A sharp sup+inf inequality for a nonlinear elliptic equation in $\mathbb{R}^{2}$. Comm. Anal. Geom. 6, 1-19 (1998)

18. Chen, C.C. and Lin, C.S.: Estimates of the conformal scalar curvature equation via the method of moving planes. Comm. Pure Appl. Math. 50, 971-1017 (1997)

19. Chen, W. and Li, C.: Classification of solutions of some nonlinear elliptic equations. Duke Math. J. 63, 615-623 (1991)

20. Chen, X.: Remarks on the existence of branch bubbles on the blowup analysis of equation $-\Delta u=e^{2 u}$ in dimension two. Comm. Anal. Geom., to appear

21. Chou, K.S. and Wan, T.Y.H.: Asymptotic radial symmetry for solutions of $\Delta u+e^{u}=0$ in a punctured disc. In: Elliptic and parabolic methods in geometry, Minneapolis, MN, 1994, Wellesley, MA: A K Peters, 1996, pp. 17-21

22. Ding, W., Jost, J., Li, J. and Wang, G.: The differential equation $\Delta u=8 \pi-8 \pi h e^{u}$ on a compact Riemann surface. Asian J. Math. 1, 230-248 (1997)

23. Ding, W., Jost, J., Li, J. and Wang, G.: An analysis of the two-vortex case in the Chern-Simons Higgs model. Calc. Var. 7, 87-97 (1998)

24. Dunne, G.: Self-Dual Chern-Simons Theories. Lecture notes in Physics, New Series M 36, New York: Springer, 1996

25. Gidas, B., Ni, W.M. and Nirenberg, L.: Symmetry and related properties via the maximum principle. Commun. Math. Phys. 68, 209-243 (1979)

26. Gidas, B., Ni, W.M. and Nirenberg, L.: Symmetry of positive solutions of nonlinear elliptic equations in $\mathbb{R}^{n}$. Math. Anal. and Applications, Part A, Advances in Math. Suppl. Studies 7A, (ed. L. Nachbin), London-New York: Academic Pr., 1981, pp. 369-402

27. Hong, J., Kim, Y. and Pac, P.Y.: Multivortex solutions of the Abelian Chern Simons theory. Phys. Rev. Lett. 64, 2230-2233 (1990)

28. Jackiw, R. and Weinberg, E.J.: Selfdual Chern Simons vortices. Phys. Rev. Lett. 64 2234-2237 (1990),

29. Kazdan, J. and Warner, F.: Curvature functions for compact 2-manifolds. Ann. of Math. 99, 14-47 (1974)

30. Kiessling, M.K.H.: Statistical mechanics of classical particles with logarithmic interaction. Comm. Pure Appl. Math. 46, 27-56 (1993)

31. Li, Y.Y.: Prescribing scalar curvature on $S^{n}$ and related problems, Part II: Existence and compactness. Comm. Pure Appl. Math. 49, 541-597 (1996)

32. Li, Y.Y. and Shafrir, I.: Blow-up analysis for solutions of $-\Delta u=V e^{u}$ in dimension two. Indiana Univ. Math. J. 43, 1255-1270 (1994)

33. Moser, J.: On a nonlinear problem in differential geometry. In: Dynamical Systems (M. Peixoto, ed.), New York: Academic Press, 1973, pp. 273-280

34. Nirenberg, L.: Topics in Nonlinear Functional Analysis. Lecture Notes, Courant Institute, New York University, 1974

35. Struwe, M. and Tarantello, G.: On multivortex solutions in Chern-Simons Gauge theory. Boll. Unione Mat. Ital. Sez. B Artic. Ric. Mat. 8 1, 109-121 (1998)

36. Schoen, R.: Courses at Stanford University (1988) and New York University (1989), unpublished

37. Serrin, J.: A symmetry problem in potential theory. Arch. Rat. Mech. Anal. 43, 304-318 (1971)

38. Spruck, J. and Yang, Y.: Topological solutions in the self-dual Chern-Simons theory: Existence and approximation. Ann. Inst. H. Poincaré Anal. Non Linéaire 12, 75-97 (1995) 
39. Tarantello, G.: Multiple condensate solutions for the Chern-Simons-Higgs theory. J. Math. Phys. 37, 3769-3796 (1996)

40. Taubes, C.H.: Arbitrary $N$-vortex solutions to the first order Ginzburg-Landau equation. Commun. Math. Phys. 72, 277-292 (1980)

41. Taubes, C.H.: On the equivalence of the first and second order equations for gauge theories. Commun. Math. Phys. 75, 207-227 (1980)

Communicated by J. L. Lebowitz 